\title{
Evaluation of the clinical benefits of adjuvant capecitabine monotherapy in elderly women with breast cancer: A retrospective study
}

\author{
YAN-SHUANG LI ${ }^{1}$, QING YANG ${ }^{2}$, MING $^{2}{ }^{2}$ and JI-YU LI ${ }^{2}$ \\ ${ }^{1}$ Department of Neurology, Jinan Central Hospital Affiliated to Shandong University, Jinan, \\ Shandong 250013; ${ }^{2}$ Department of Breast and Thyroid Surgery, Shandong Provincial Hospital \\ Affiliated to Shandong University, Jinan, Shandong 250021, P.R. China
}

Received August 5, 2016; Accepted April 3, 2017

DOI: $10.3892 / \mathrm{mco} .2017 .1351$

\begin{abstract}
Capecitabine is orally administered and may be safely and conveniently used in patients with cancer. The antitumor activity of capecitabine in breast cancer was mostly demonstrated in the salvage therapy setting, whereas the effect of adjuvant capecitabine monotherapy in breast cancer remains unclear. The aim of the present study was to evaluate adjuvant capecitabine monotherapy in elderly women with breast cancer. A total of 251 patients were enrolled and survival was compared between elderly breast cancer patients who received adjuvant capecitabine monotherapy and those who received no chemotherapy. Cancer-specific and disease-free survival curves were compared using log-rank tests and survival curves were calculated using the Kaplan-Meier method. Multivariate analyses were conducted using Cox's proportional hazard regression model. There was no significant difference between the clinicopathological characteristics, including age, tumor size, lymph node status, histological grade and hormone status, between patients in the two groups. The breast cancer-specific survival rate was $89.3 \%$ in the capecitabine monotherapy group vs. $81.3 \%$ in the no chemotherapy group; the difference was not statistically significant $(\mathrm{P}=0.128)$. The disease-free survival rate was $81.7 \%$ in the capecitabine monotherapy group vs. $65.3 \%$ in the no chemotherapy group. Kaplan-Meier analysis indicated a longer disease-free survival in the capecitabine monotherapy group $(\mathrm{P}=0.015)$. On Cox regression analysis, capecitabine monotherapy was found to be associated with the disease-free survival rate $(\mathrm{P}=0.014$, hazard ratio $=0.500)$ but not with the cancer-specific survival rate $(\mathrm{P}=0.181)$. The
\end{abstract}

Correspondence to: Dr Ji-Yu Li, Department of Breast and Thyroid Surgery, Shandong Provincial Hospital Affiliated to Shandong University, 324 Jingwu-Weiqi Road, Jinan, Shandong 250021, P.R. China

E-mail: bigli2004@163.com

Key words: breast neoplasm, capecitabine, monotherapy, chemotherapy, elderly women adverse events of capecitabine monotherapy were recorded and there was no chemotherapy interruption due to severe adverse reactions. Therefore, adjuvant capecitabine monotherapy in elderly women with breast cancer is a safe and effective option, as well as a viable alternative for elderly breast cancer patients who refuse standard adjuvant therapy.

\section{Introduction}

Breast cancer is one of the most common malignant tumors in women worldwide (1). The treatment generally includes surgery, radiation and chemotherapy. Chemotherapy plays an important role in the multidisciplinary treatment of breast cancer. A number of clinical trials have demonstrated that adjuvant chemotherapy may improve survival among women with breast cancer (2-4). Anthracyclines and taxanes are usually included in the polychemotherapy used to treat breast cancer, but their side effects, such as neutropenia, vomiting and diarrhea, may be difficult for elderly women to tolerate. Due to these severe side effects, several elderly patients refuse to receive standard polychemotherapy intravenously. Patients occasionally prefer more easily tolerated oral chemotherapeutic agents as an alternative to standard adjuvant chemotherapy. Furthermore, elderly women with breast cancer often suffer from comorbidities, such as hypertension, diabetes and heart disease, and they are frequently excluded from clinical trials. As a result, breast cancer in elderly patients is not always treated according to the guidelines. Oncologists occasionally administer oral chemotherapeutic agents to elderly patients to replace the standard intravenous adjuvant chemotherapy. Capecitabine, an orally administered fluoropyrimidine carbamate, has a safe toxicity profile and is often administered to elderly patients as single-agent adjuvant chemotherapy (5). Although the antitumor activity of capecitabine in locally advanced or metastatic breast has been reported, whether elderly patients with breast cancer benefit from adjuvant capecitabine monotherapy remains unclear. The aim of the present study was to retrospectively analyze clinical data from our department to evaluate the clinical benefits of adjuvant capecitabine monotherapy in elderly women with breast cancer. 


\section{Patients and methods}

Patients. This retrospective cohort involved 251 primary invasive breast cancer patients aged $\geq 60$ years who underwent surgery at the Department of Breast and Thyroid Surgery of the Shandong Provincial Hospital Affiliated to Shandong University (Jinan, China) between June 2001 and June 2013. A total of 147 patients received no chemotherapy and 104 patients received oral capecitabine monotherapy (Xeloda ${ }^{\circledR}$, Roche Pharma AG, Grenzach-Wyhlen, Germany).

Patients who received any form of chemotherapy other than capecitabine were not included, and patients who received neoadjuvant chemotherapy or endocrine therapy were also excluded. Treatment of radiotherapy or adjuvant endocrine therapy was not considered as an exclusion criterion. No patients received adjuvant trastuzumab therapy. All the patients were menopausal. The majority of the patients underwent modified radical mastectomy. The clinical and pathological characteristics of the patients are summarized in Table I.

In the capecitabine monotherapy group, the patients were treated with capecitabine $1,250 \mathrm{mg} / \mathrm{m}^{2}$ twice daily for 14 days every 21 days as one cycle. Six cycles were planned. Toxic reactions were assessed according to the Common Terminology Criteria for Adverse Events, version 4.0 (https://evs.nci.nih.gov/ftp1/CTCAE/CTCAE_ 4.03_2010-06-14_QuickReference_8.5x11.pdf). All the patients with hormone receptor-positive tumors in both groups received endocrine therapy regularly. The study protocol was approved by the Ethics Committee of the Shandong Provincial Hospital and written informed consent was obtained from the participants for their clinical records to be used in this study.

Follow-up. The adverse events of capecitabine monotherapy were recorded every 8 weeks during outpatient visits. Follow-up information regarding tumor recurrence and survival status after chemotherapy was collected through personal contact with the patients by routine letters, telephone visits, or a questionnaire, conducted at the Shandong Provincial Hospital every 3 months during the first 2 years after surgery, every 6 months during the next 2 years, and once a year thereafter.

Statistical analysis. A Chi-squared test was used to compare clinicopathological characteristics between patients treated with capecitabine monotherapy or without chemotherapy. Cancer-specific survival was defined as the time from the first diagnosis of primary breast cancer to death caused by the cancer, and disease-free survival was defined as the time from the first diagnosis of primary breast cancer to local recurrence or metastasis. Cancer-specific and disease-free survival curves were compared using log-rank tests. Survival curves were calculated using the Kaplan-Meier method. Multivariate analyses were conducted using Cox's proportional hazard regression model, which included variables such as tumor size, lymph node status and nuclear grade in the multivariate analysis model. Hazard ratios (HR) were calculated with their $95 \%$ confidence intervals. P-values $<0.05$ were considered to indicate statistically significant differences. Statistical analysis was performed using IBM SPSS software, version 20 (IBM Corp., Armonk, NY, USA).

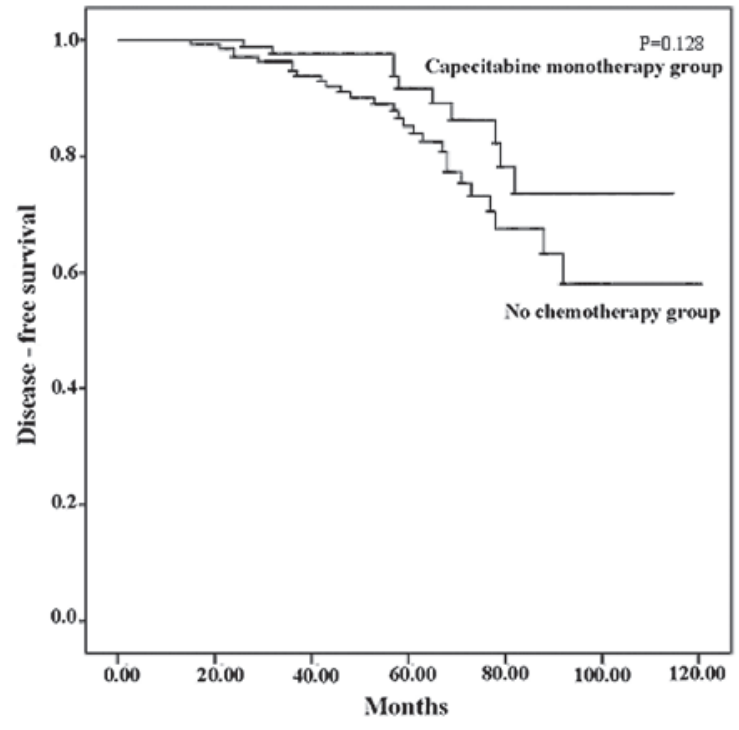

Figure 1. Kaplan-Meier curves for cancer-specific survival. Although the cancer-specific survival rate in the capecitabine monotherapy group was higher, the difference between the two groups was not statistically significant $(\mathrm{P}=0.128)$.

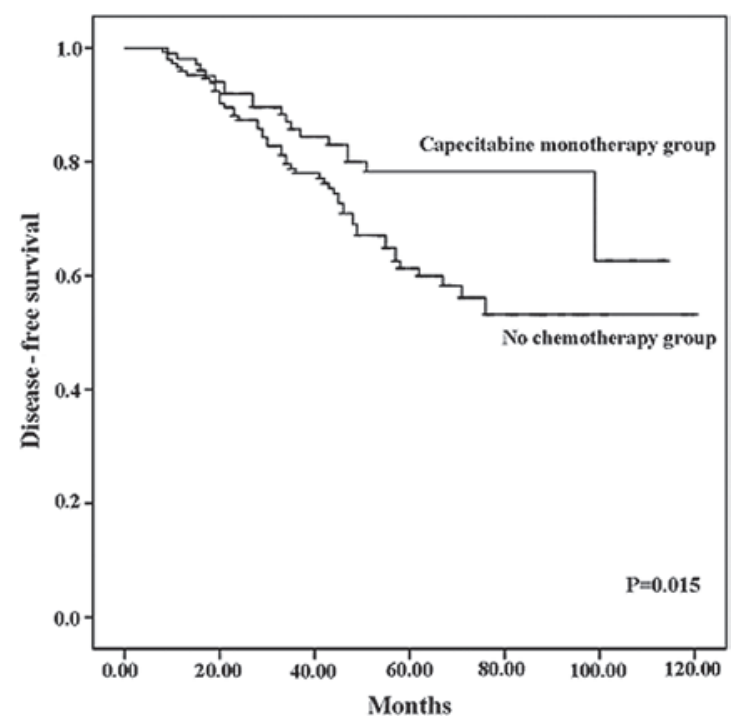

Figure 2. Kaplan-Meier curves for disease-free survival. Capecitabine monotherapy significantly reduced the recurrence rate $(\mathrm{P}=0.015)$.

\section{Results}

Patient characteristics. A total of 251 patients met the inclusion criteria of this retrospective study, with 147 patients assigned to the no chemotherapy group and 104 to the capecitabine monotherapy group. The clinical and pathological characteristics of all the patients are summarized in Table I. There were no significant differences in prognostic factors, such as age, grade, number of positive lymph nodes and human epidermal growth factor receptor (HER)2 status, between the capecitabine monotherapy and the no chemotherapy groups $(\mathrm{P}>0.05)$. Estrogen receptor and progesterone receptor status were also similar between the two groups $(\mathrm{P}>0.05)$.

Cancer-specific and disease-free survival. In the capecitabine monotherapy group, the breast cancer-specific survival rate 
Table I. Clinicopathological characteristics of the patients.

\begin{tabular}{|c|c|c|c|}
\hline Characteristics & No chemotherapy $(n=147)$ & Capecitabine monotherapy $(n=104)$ & P-value \\
\hline Mean age (years) & 67.9 & 66.9 & 0.14 \\
\hline Tumor size (cm) & & & 0.56 \\
\hline$\leq 2$ & 44 & 30 & \\
\hline$>2$ to $\leq 5$ & 75 & 59 & \\
\hline$>5$ & 28 & 15 & \\
\hline Grade & & & 0.85 \\
\hline I & 9 & 8 & \\
\hline II & 55 & 40 & \\
\hline III & 73 & 49 & \\
\hline Not available & 10 & 7 & \\
\hline Positive lymph nodes (n) & & & 0.89 \\
\hline 0 & 83 & 57 & \\
\hline $1-3$ & 35 & 29 & \\
\hline $4-9$ & 18 & 11 & \\
\hline$\geq 10$ & 11 & 7 & \\
\hline ER status (n) & & & 0.31 \\
\hline Positive & 103 & 72 & \\
\hline Negative & 44 & 32 & \\
\hline PR status (n) & & & 0.87 \\
\hline Positive & 92 & 64 & \\
\hline Negative & 55 & 40 & \\
\hline HER2 status (n) & & & 0.74 \\
\hline Positive & 15 & 12 & \\
\hline Negative & 132 & 92 & \\
\hline Surgery patterns (n) & & & 0.97 \\
\hline Modified radical mastectomy & 132 & 94 & \\
\hline Simple mastectomy & 11 & 7 & \\
\hline Breastconserving surgery & 4 & 3 & \\
\hline
\end{tabular}

ER, estrogen receptor; PR, progesterone receptor; HER2, human epidermal growth factor receptor 2.

was $89.3 \%$, with a median follow-up of 54 months (range, 13-114 months). The breast cancer-specific survival rate in the no chemotherapy group was $81.3 \%$, with a median follow-up of 57 months (range, 10-120 months). One patient in the capecitabine monotherapy group and 3 patients in the no chemotherapy group succumbed to other causes. Although the cancer-specific survival rate in the capecitabine monotherapy group was higher, there were still no statistically significant differences between the two groups in this respect $(\mathrm{P}=0.128)$. The Kaplan-Meier curves for cancer specific survival are shown in Fig. 1.

The disease-free survival rate was $81.7 \%$ in the capecitabine monotherapy group and $65.3 \%$ in the no chemotherapy group. Capecitabine monotherapy significantly reduced the recurrence rate $(\mathrm{P}=0.015)$. The Kaplan-Meier curves for disease-free survival are shown in Fig. 2. Recurrence occurred in 19 patients in the capecitabine monotherapy group and 51 in the no chemotherapy group. The details on recurrence sites are presented in Table II.

Multivariate survival analysis. Based on the Cox regression analysis, capecitabine monotherapy was a significant
Table II. Details of recurrence sites in the two groups.

\begin{tabular}{lcc}
\hline Recurrence site & $\begin{array}{c}\text { No } \\
\text { chemotherapy }(\mathrm{n}=51)\end{array}$ & $\begin{array}{c}\text { Capecitabine } \\
\text { monotherapy }(\mathrm{n}=19)\end{array}$ \\
\hline Locoregional & 21 & 8 \\
Lung & 19 & 7 \\
Liver & 12 & 5 \\
Bone & 16 & 6 \\
Others & 4 & 1 \\
\hline
\end{tabular}

independent predictive factor for disease-free survival rate $(\mathrm{P}=0.014, \mathrm{HR}=0.500$, 95\% CI: 0.288-0.867; Table III), although it was not an independent factor for cancerspecific survival rate $(\mathrm{P}=0.181$, Table IV).

Adverse events and comorbidities. The adverse events of capecitabine monotherapy were recorded. Hand-foot syndrome in the capecitabine monotherapy group mainly occurred after 
Table III. Multivariate analysis of diseasefree survival by Cox proportional hazards models.

\begin{tabular}{lcc}
\hline Variables & P-value & Hazard ratio (95\% CI) \\
\hline Treatment (capecitabine monotherapy vs. no chemotherapy) & 0.014 & $0.500(0.288-0.867)$ \\
Tumor size ( $\leq 2$ vs. $>2$ cm) & 0.034 & $1.512(1.032-2.215)$ \\
Nuclear grade (I or II vs. III) & 0.233 & - \\
Axillary node status (positive vs. negative) & 0.000 & $2.086(1.605-2.713)$ \\
HER2 status (positive vs. negative) & 0.000 & $3.807(2.123-6.826)$ \\
ER status (positive vs. negative) & 0.381 & - \\
PR status (positive vs. negative) & 0.587 & - \\
\hline
\end{tabular}

ER, estrogen receptor; PR, progesterone receptor; HER2, human epidermal growth factor receptor 2.

Table IV. Multivariate analysis of cancerspecific survival by Cox proportional hazards models

\begin{tabular}{lcc}
\hline Variables & P-value & Hazard ratio (95\% CI) \\
\hline Treatment (capecitabine monotherapy vs. no chemotherapy) & 0.181 & - \\
Tumor size ( $\leq 2$ vs. $>2 \mathrm{~cm}$ ) & 0.648 & - \\
Nuclear grade (I or II vs. III) & 0.201 & - \\
Axilla status (positive vs. negative) & 0.000 & $2.785(1.924-4.031)$ \\
HER2 status (positive vs. negative) & 0.030 & $2.412(1.092-5.332)$ \\
ER status (positive vs. negative) & 0.323 & - \\
PR status (positive vs. negative) & 0.370 & - \\
\hline
\end{tabular}

ER, estrogen receptor; PR, progesterone receptor; HER2, human epidermal growth factor receptor 2.

3 treatment cycles. Among the capecitabine monotherapy group, 10 patients had grade $\geq 3$ serious hand-foot syndrome, among whom 2 aborted the chemotherapy after 3 and 4 cycles. Other adverse events, including anemia, neutropenia, nausea, vomiting, diarrhea and liver injury, are summarized in Table V. Therefore, capecitabine appeared to be well-tolerated by elderly women with breast cancer in the present study.

The patients' comorbidities are summarized in Table VI. The incidence of hypertension, cardiovascular disease and cerebral vascular disease was higher in the no chemotherapy group compared with the capecitabine monotherapy group. The percentage of patients with $\geq 2$ comorbidities was also higher in the no chemotherapy group. Comorbidities significantly affected the patients' decision to receive chemotherapy.

\section{Discussion}

Capecitabine is an orally administered chemotherapeutic agent that was licensed to be used as monotherapy for the treatment of patients with locally advanced or metastatic breast cancer following failure of previous anthracycline or taxane-containing chemotherapy (6). The efficacy of capecitabine in metastatic or locally advanced breast cancer has been evaluated in various phase II/III clinical trials. The response rate of metastatic breast cancer to capecitabine monotherapy is $\sim 30 \%(6,7)$. Capecitabine clearly improved the survival rate in second- and later-line salvage chemotherapy (6-10), and its efficacy has been also proven in
Table V. Grade 3, 4 or 5 adverse eventsa in the capecitabine monotherapy group.

\begin{tabular}{lc}
\hline Adverse events & No. of patients (\%) \\
\hline Hematological & \\
Anemia & $2(1.9)$ \\
Neutropenia & $5(4.8)$ \\
Febrile neutropenia & $0(0.0)$ \\
Gastrointestinal reactions & \\
Nausea & $2(1.9)$ \\
Vomiting & $3(2.9)$ \\
Diarrhea & $3(2.9)$ \\
Liver injury & \\
ALT increased & $2(1.9)$ \\
AST increased & $4(3.8)$ \\
Handfoot skin reaction & $10(9.6)$ \\
\hline
\end{tabular}

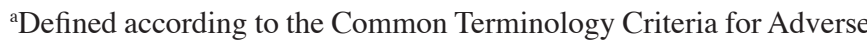
Events, version 4.0. ALT, alanine transaminase; AST, aspartate transaminase.

the first-line treatment of metastatic breast cancer (11-14). Kamal et al observed similar survival between capecitabine monotherapy and single-agent taxane as a first-line therapy for metastatic breast cancer (15). 
Table VI. Comorbidities of patients in the two groups.

\begin{tabular}{lccc}
\hline Comorbidities & No chemotherapy $(\mathrm{n}=147)$ & Capecitabine monotherapy $(\mathrm{n}=104)$ & P-value \\
\hline Hypertension & 71 & 30 & 0.003 \\
Diabetes & 41 & 18 & 0.069 \\
Cardiovascular disease & 38 & 12 & 0.006 \\
Cerebrovascular disease & 27 & 7 & 0.008 \\
$\geq 2$ comorbidities & 28 & 4 & $2.283 \times 10^{-4}$ \\
No comorbidity & 19 & 41 & $2.085 \times 10^{-6}$ \\
\hline
\end{tabular}

Capecitabine has also been used was neoadjuvant chemotherapy for breast cancer. Arowolo et al reported that capecitabine monotherapy achieved good overall response rates with manageable toxicity when administered as neoadjuvant chemotherapy to 16 patients with locally advanced breast cancer (16). In a phase II study by Tolaney et al, 3 patients exhibited a complete clinical response after 4 cycles of capecitabine monotherapy and 8 patients exhibited a partial clinical response among 24 women with operable hormone receptor-positive breast cancer (17). Li et al reviewed the randomized controlled trials that included capecitabine in the neoadjuvant chemotherapy for breast cancer and concluded that adding capecitabine to neoadjuvant chemotherapy was unlikely to improve the outcomes in breast cancer patients without distant metastases (18).

The antitumor activity of capecitabine in metastatic or locally advanced breast cancer makes it a potential alternative to standard adjuvant chemotherapy for breast cancer. While capecitabine demonstrated a poor efficacy as neoadjuvant therapy for breast cancer, its efficacy in the adjuvant setting has not been fully elucidated. In fact, it is not currently rare to use adjuvant capecitabine monotherapy in elderly women with breast cancer who are unable to undergo standard chemotherapy in China. However, there is little evidence to support its effectiveness. A meta-analysis by Jiang et al demonstrated that disease-free survival significantly improved following addition of capecitabine to an anthracycline-taxane-based adjuvant chemotherapy in patients with high-risk early breast cancer (19). The final results of the FinXX trial indicated that the addition of capecitabine significantly improved breast cancer-specific survival, although not the recurrence-free survival, in early breast cancer (20). A small retrospective study involving elderly patients with stage IIa breast cancer by $\mathrm{Hu}$ et al reported that the patients who underwent adjuvant capecitabine monotherapy had a similar overall survival compared with those who received traditional cyclophosphamide/epirubicin/5-fluorouracil (CAF regimen) chemotherapy, but fewer adverse reactions (21). They compared the overall survival of the two groups by Kaplan-Meier analysis, a univariate method; however, multivariate survival analysis is required to investigate whether capecitabine monotherapy is an independent factor that affects survival. A randomized clinical trial by Muss et al revealed that standard adjuvant chemotherapy was superior to capecitabine monotherapy in elderly patients with early-stage breast cancer (22). The standard adjuvant chemotherapy included CMF cyclophosphamide, methotrexate and fluorouracil (CMF regimen) and doxorubicin/cyclophosphamide (AC regimen). The relapse-free survival rate was $68 \%$ in the capecitabine monotherapy group vs. $85 \%$ in the standard chemotherapy group $(\mathrm{P}<0.001)$, and the overall survival rate was 86 vs. $91 \%(\mathrm{P}=0.02)$. However, whether elderly women with breast cancer benefit from adjuvant capecitabine monotherapy remains unclear.

To the best of our knowledge, the present retrospective study was the first to demonstrate that adjuvant capecitabine monotherapy significantly reduced recurrence or metastasis in elderly patients with breast cancer. The disease-free survival rate was $81.7 \%$ in the capecitabine monotherapy group and $65.3 \%$ in the no chemotherapy group $(\mathrm{P}=0.015)$. In the Kaplan-Meier analysis, the disease-free survival curve of the capecitabine monotherapy group was superior to that of the no chemotherapy group (Fig. 1). The breast cancer-specific survival rate of the capecitabine monotherapy group was $89.3 \%$ with a median follow-up of 54 months, whereas it was $81.3 \%$ with a median follow-up of 57 months in the no chemotherapy group. The cancer-specific survival curve of the capecitabine monotherapy group was superior to that of the no chemotherapy group (Fig. 2), although the difference was not statistically significant $(\mathrm{P}=0.128)$. The results of the Cox regression analysis demonstrated that capecitabine was an independent predictive factor for disease-free survival, but not for cancer-specific survival. Adjuvant capecitabine monotherapy did not improve the cancer-specific survival rate. This finding may be due to the short median follow-up duration. Positive results for cancer-specific survival may be obtained in future analyses.

The present study included elderly patients aged $\geq 60$ years, according to the definition of elderly individuals in the Law of the People's Republic of China on Protection of the Rights and Interests of the Elderly. Furthermore, only a limited number of patients aged $<60$ years received adjuvant capecitabine monotherapy in our department.

Adjuvant trastuzumab therapy was shown to significantly improve disease-free and overall survival in patients with HER2-positive early breast cancer (23). None of the 27 HER2-positive patients in this retrospective cohort received adjuvant trastuzumab therapy, as trastuzumab is not covered by health insurance in China, and the majority of elderly patients cannot afford this treatment.

Capecitabine is a fluoropyrimidine carbamate that achieves higher intratumoral levels with a lower toxicity compared with 5-fluorouracil (24). Capecitabine was considered as a chemotherapeutic agent that was safe to use in patients with liver or renal disease. In the present study, $\sim 60 \%$ of patients in the capecitabine monotherapy group had comorbidities, 
such as hypertension, diabetes, cerebrovascular disease and cardiovascular disease. Severe adverse reactions were rare, and the most common adverse event was hand-foot syndrome, which occurred in 10 patients. Other adverse events, including hematological adverse events, gastrointestinal reactions and liver injury, were tolerable. None of the patients interrupted the chemotherapy due to a severe adverse reaction. Thus, capecitabine monotherapy was safe to for elderly patients, even those with comorbidities. The comorbidities were compared between different groups (Table VI) and were found to be more frequent in the no chemotherapy group. Although capecitabine monotherapy was safe, the comorbidities of the patients affected their decision to receive this orally administered chemotherapeutic agent.

The present study has some inevitable limitations due to its retrospective nature. A prospective, randomized control study has been designed and will be initiated in the near future. Additionally, the effect of different endocrine therapy agents that were administered to patients was not evaluated.

In summary, adjuvant capecitabine monotherapy was found to be effective in elderly patients with breast cancer and its side effects were manageable. Capecitabine monotherapy is a good alternative for elderly breast cancer patients who refuse standard adjuvant therapy. Our findings suggest that adjuvant capecitabine monotherapy in elderly women with breast cancer was a safe and effective treatment option.

\section{Acknowledgements}

The authors would like to thank the participating patients for their willingness to cooperate. We also greatly appreciate the assistance of American Journal Experts (AJE) editors. This study was supported by the Key Research and Development Program of Shandong Province (grant no. 2015GSF118023) and the Projects of Medical and Health Technology Development Program in Shandong Province (grant no. 2014WS0349).

\section{References}

1. Siegel R, Ma J, Zou Z and Jemal A: Cancer statistics, 2014. CA Cancer J Clin 64: 9-29, 2014.

2. Joensuu H: Systemic chemotherapy for cancer: From weapon to treatment. Lancet Oncol 9: 304, 2008

3. Early Breast Cancer Trialists' Collaborative Group (EBCTCG): Effects of chemotherapy and hormonal therapy for early breast cancer on recurrence and 15-year survival: An overview of the randomised trials. Lancet 365: 1687-1717, 2005.

4. Berry DA, Cronin KA, Plevritis SK, Fryback DG, Clarke L, Zelen M, Mandelblatt JS, Yakovlev AY, Habbema JD and Feuer EJ Cancer Intervention and Surveillance Modeling Network (CISNET) Collaborators: Effect of screening and adjuvant therapy on mortality from breast cancer. N Engl J Med 353: 1784-1792, 2005.

5. Mikhail SE, Sun JF and Marshall JL: Safety of capecitabine: A review. Expert Opin Drug Saf 9: 831-841, 2010.

6. Blum JL: The role of capecitabine, an oral, enzymatically activated fluoropyrimidine, in the treatment of metastatic breast cancer. Oncologist 6: 56-64, 2001.

7. Blum JL, Jones SE, Buzdar AU, LoRusso PM, Kuter I, Vogel C, Osterwalder B, Burger HU, Brown CS and Griffin T: Multicenter phase II study of capecitabine in paclitaxel-refractory metastatic breast cancer. J Clin Oncol 17: 485-493, 1999.

8. Miller KD, Chap LI, Holmes FA, Cobleigh MA, Marcom PK, Fehrenbacher L, Dickler M, Overmoyer BA, Reimann JD, Sing AP, et al: Randomized phase III trial of capecitabine compared with bevacizumab plus capecitabine in patients with previously treated metastatic breast cancer. J Clin Oncol 23: 792-799, 2005
9. Talbot DC, Moiseyenko V, Van Belle S, O'Reilly SM, Alba Conejo E, Ackland S, Eisenberg P, Melnychuk D, Pienkowski T, Burger HU, et al: Randomised, phase II trial comparing oral capecitabine (Xeloda) with paclitaxel in patients with metastatic/advanced breast cancer pretreated with anthracyclines. Br J Cancer 86: 1367-1372, 2002.

10. Schott AF, Barlow WE, Albain KS, Chew HK, Wade JL III, Lanier KS, Lew DL, Hayes DF, Gralow JR, Livingston RB and Hortobagyi GN: Phase II trial of simple oral therapy with capecitabine and cyclophosphamide in patients with metastatic breast cancer: SWOG S0430. Oncologist 17: 179-187, 2012.

11. Stockler MR, Harvey VJ, Francis PA, Byrne MJ, Ackland SP, Fitzharris B, Van Hazel G, Wilcken NR, Grimison PS, Nowak AK, et al: Capecitabine versus classical cyclophosphamide, methotrexate, and fluorouracil as first-line chemotherapy for advanced breast cancer. J Clin Oncol 29: 4498-4504, 2011.

12. Kaufmann M, Maass N, Costa SD, Schneeweiss A, Loibl S, Sütterlin MW, Schrader I, Gerber B, Bauer W, Wiest W, et al: First-line therapy with moderate dose capecitabine in metastatic breast cancer is safe and active: Results of the MONICA trial. Eur J Cancer 46: 3184-3191, 2010.

13. Oshaughnessy JA, Blum J, Moiseyenko V, Jones SE, Miles D, Bell D, Rosso R, Mauriac L, Osterwalder B, Burger HU and Laws S: Randomized, open-label, phase II trial of oral capecitabine (Xeloda) vs. a reference arm of intravenous CMF (cyclophosphamide, methotrexate and 5-fluorouracil) as first-line therapy for advanced/metastatic breast cancer. Ann Oncol 12: 1247-1254, 2001.

14. Blum JL, Barrios CH, Feldman N, Verma S, McKenna EF, Lee LF, Scotto N and Gralow J: Pooled analysis of individual patient data from capecitabine monotherapy clinical trials in locally advanced or metastatic breast cancer. Breast Cancer Res Treat 136: 777-788, 2012.

15. Kamal AH, Camacho F, Anderson R, Wei W, Balkrishnan R and Kimmick G: Similar survival with single-agent capecitabine or taxane in first-line therapy for metastatic breast cancer. Breast Cancer Res Treat 134: 371-378, 2012.

16. Arowolo OA, Njiaju UO, Ogundiran TO, Abidoye O, Lawal OO, Obajimi M, Adetiloye AV, Im HK, Akinkuolie AA, Oluwasola A, et al: Neo-adjuvant capecitabine chemotherapy in women with newly diagnosed locally advanced breast cancer in a resource-poor setting (Nigeria): Efficacy and safety in a phase II feasibility study. Breast J 19: 470-477, 2013.

17. Tolaney SM, Jeong J, Guo H, Brock J, Morganstern D, Come SE, Golshan M, Bellon J, Winer EP and Krop IE: A phase II study of preoperative capecitabine in women with operable hormone receptor positive breast cancer. Cancer Med 3: 293-299, 2014.

18. Li Q, Jiang Y, Wei W, Yang H and Liu J: Clinical efficacy of including capecitabine in neoadjuvant chemotherapy for breast cancer: A systematic review and meta-analysis of randomized controlled trials. PLoS One 8: e53403, 2013.

19. Jiang Y, Yin W, Zhou L, Yan T, Zhou Q, Du Y, Shen Z, Shao $\mathrm{Z}$ and $\mathrm{Lu} \mathrm{J}$ : First efficacy results of capecitabine with anthracycline- and taxane-based adjuvant therapy in high-risk early breast cancer: A meta-analysis. PLoS One 7: e32474, 2012.

20. Joensuu H, Kellokumpu-Lehtinen PL, Huovinen R, Jukkola-Vuorinen A, Tanner M, Kokko R, Ahlgren J, Auvinen P, Paija O, Helle L, et al: Adjuvant capecitabine, docetaxel, cyclophosphamide, and epirubicin for early breast cancer: Final analysis of the randomized FinXX trial. J Clin Oncol 30: 11-18, 2012.

21. Hu W, Shi J, Sheng Y, Li L, Su D and Wang CK: Clinical study of adjuvant capecitabine monotherapy in Chinese elderly patients (aged 55-70) with stage IIa breast cancer. Onkologie 33: 433-436, 2010.

22. Muss HB, Berry DA, Cirrincione CT, Theodoulou M, Mauer AM, Kornblith AB, Partridge AH, Dressler LG, Cohen HJ, Becker HP, et al: Adjuvant chemotherapy in older women with early-stage breast cancer. N Engl J Med 360: 2055-2065, 2009.

23. Gianni L, Dafni U, Gelber RD, Azambuja E, Muehlbauer S, Goldhirsch A, Untch M, Smith I, Baselga J, Jackisch C, et al: Treatment with trastuzumab for 1 year after adjuvant chemotherapy in patients with HER2-positive early breast cancer: A 4-year follow-up of a randomised controlled trial. Lancet Oncol 12: 236-244, 2011.

24. Nole F, Catania C, Munzone E, Rocca A, Verri E, Sanna G, Ascione G, Adamoli L, Zampino MG, Minchella I and Goldhirsch A: Capecitabine/vinorelbine: An effective and well-tolerated regimen for women with pretreated advanced-stage breast cancer. Clin Breast Cancer 6: 518-524, 2006. 